\title{
Impact of kinetics on the growth of GaN on graphene by plasma-assisted molecular beam epitaxy
}

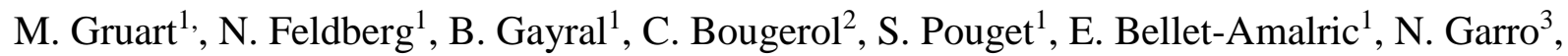
A. Cros ${ }^{3}$, H. Okuno ${ }^{4}$ and B. Daudin ${ }^{1}$

${ }^{1}$ Univ. Grenoble-Alpes, CEA-IRIG, PHELIQS, 17 av. des Martyrs, 38000 Grenoble, France.

${ }^{2}$ Univ. Grenoble-Alpes, CNRS-Institut Néel, 25 av. des Martyrs, 38000 Grenoble, France.

${ }^{3}$ Institute of Materials Science (ICMUV), Universidad de Valencia, P.O. Box 22085, Valencia, Spain

${ }^{4}$ Univ. Grenoble-Alpes, CEA-IRIG, MEM, 17 av. des Martyrs, 38000 Grenoble, France.

Corresponding author: bruno.daudin@cea.fr

\begin{abstract}
The growth of GaN on graphene by molecular beam epitaxy was investigated. The most stable epitaxial relationship, i.e. [00.1]-oriented grains, is obtained at high temperature and Nrich conditions, which match those for nanowire growth. Alternatively, at moderate temperature and Ga-rich conditions, several metastable orientations are observed at the nucleation stage, which evolve preferentially towards [00.1]-oriented grains. The dependence of the nucleation regime on growth conditions was assigned to Ga adatom kinetics. This statement is consistent with the calculated graphene/GaN in-plane lattice coincidence and supported by a combination of transmission electron microscopy, X-ray diffraction, photoluminescence, and Raman spectroscopy experiments.
\end{abstract}




\section{Introduction}

Despite the continuous progress in epitaxial growth techniques, the lack of latticematched substrates for the growth of III-nitride semiconductor materials is still an obstacle to the realization of optimized devices. However strain induced by lattice mismatch can be significantly relaxed if the epitaxial growth is achieved through van der Waals (VdW) interactions [1]. Indeed, in the absence of dangling bonds, the growing crystal will not form chemical bonds with the substrate. Hence, it is expected that the elastic strain will be minimized or even completely suppressed, leading to the formation of a material with a high crystallographic quality. This approach, namely $\mathrm{VdW}$ epitaxy, has already demonstrated to overcome the difficulties of heteroepitaxy, with a particular success in the case of danglingbond-free two-dimensional (2D) materials, such as transition metal dichalcogenides [2, 3]. As concerns III-N materials, layers of AlN [4, 5] as well as GaN nanowires (NWs) [6-9] and GaN microstructures $[10,11]$ were grown on graphene by hydride vapor phase epitaxy (HVPE), molecular beam epitaxy (MBE) and metalorganic chemical vapor deposition (MOCVD) techniques. This successful new route to the controlled epitaxial growth of III-N materials has led to the realization of transferable devices [7, 12]. While there was a marked interest in demonstrating this new approach to III-nitride growth, fundamental aspects such as $\mathrm{GaN}$ nucleation on graphene or the effect of the underlying supporting substrate are not clear yet. In the case of graphene/SiC, the nucleation of AlN and GaN grown by MOCVD was shown to be influenced by step edges and graphene defect sites due to enhanced chemical reactivity [13]. Recently it was reported that the exposure of graphene to plasmas or atomic beams can lead to interesting modifications: when graphene/SiC substrate is exposed to $\mathrm{Ga}$ or In a metallic bilayer forms at the interface between graphene and $\mathrm{SiC}$ surface. Also, exposure to $\mathrm{N}$ plasma leads to homogeneization of graphene doping and strain state. [14]. In this context, it appears necessary to investigate the interplay between metallic/ $\mathrm{N}$ fluxes and graphene during the first stages of 
the epitaxial growth of GaN by MBE. Accordingly the purpose of the present study is to thoroughly investigate the GaN nucleation mechanisms, paying special attention to detailed growth conditions, namely, temperature and $\mathrm{Ga}$ active $\mathrm{N}$ flux ratio. We have found that $\mathrm{GaN}$ nucleation on graphene is closely linked to the graphene/GaN in-plane lattice coincidence, in agreement with observations on other materials such as $\mathrm{CdS}[15]$ and $\mathrm{Sb}_{2} \mathrm{Te}_{3}[16,17]$ for which growth is found to be governed by the loose epitaxial relationships inferred from the coincidence lattice of atomic positions between graphene and the epilayer.

\section{Results and discussion}

Two different kinds of graphene were used as a substrate for GaN growth in order to investigate the role of subjacent substrate, either amorphous or crystalline. The first one is commercially available and consists of graphene grown by CVD on copper and transferred on $\mathrm{Si}(100)$ covered with $300 \mathrm{~nm}$ of thermal $\mathrm{SiO}_{2}\left(\mathrm{Gr} / \mathrm{SiO}_{2}\right)$ [18]. This monolayer graphene is polycrystalline with grain size up to $10 \mu \mathrm{m}$. It presents graphene bilayer defects (2LG), typically $1 \mu \mathrm{m}$ in size, and folds due to the transfer process. The second substrate is graphene grown by propane-hydrogen $\mathrm{CVD}$ on $\mathrm{SiC}-6 \mathrm{H}(\mathrm{Gr} / \mathrm{SiC})$. It consists of one epitaxial graphene layer deposited on a transition/buffer layer, namely one monolayer of carbon chemically bonded to SiC surface $[19,20]$, with occasional 2LG defects of much smaller size. Both substrates were washed with organic solvents and then bonded with indium on a molybdenum sample holder before introduction in the MBE machine. The temperature was measured using a thermocouple facing the rear side of the sample holder. After a two-step annealing $\left(30\right.$ minutes at $250^{\circ} \mathrm{C}$ to eliminate solvent residues followed by 10 minutes at $750^{\circ} \mathrm{C}$ ), $\mathrm{GaN}$ growth was achieved by using plasma assisted MBE (PA-MBE). The growth temperature was varied between $750^{\circ} \mathrm{C}$ and $800^{\circ} \mathrm{C}$. Stoichiometric or slightly metal-rich conditions were used for growing layers, while nitrogen-rich conditions were used for nanowires (NWs). Growth was monitored in situ by 
reflection high-energy electron diffraction (RHEED). The growth conditions of the different samples are listed in table 1.

\begin{tabular}{|c|c|c|c|c|c|c|}
\hline Name & $\mathrm{a}$ & $\mathrm{A}$ & $\mathrm{b}$ & $\mathrm{B}$ & $\mathrm{NW} 1$ & $\mathrm{NW} 2$ \\
\hline Substrate & $\mathrm{Gr} / \mathrm{SiO}_{2}$ & $\mathrm{Gr} / \mathrm{SiO}_{2}$ & $\mathrm{Gr} / \mathrm{SiC}$ & $\mathrm{Gr} / \mathrm{SiC}$ & $\mathrm{Gr} / \mathrm{SiO}_{2}$ & $\mathrm{Gr} / \mathrm{SiC}$ \\
\hline $\mathrm{T}_{\mathrm{G}}\left({ }^{\circ} \mathrm{C}\right)$ & 740 & 780 & 740 & 740 & 800 & 750 \\
\hline $\mathrm{Ga} / \mathrm{N}$ flux ratio & $\geq 1$ & $\geq 1$ & $\geq 1$ & $\geq 1$ & 0.35 & 0.35 \\
\hline Thickness/length & seeds & $370 \mathrm{~nm}$ & seeds & $520 \mathrm{~nm}$ & $290 \mathrm{~nm}$ & $270 \mathrm{~nm}$ \\
\hline
\end{tabular}

Table 1: List of samples and growth conditions

In a preliminary set of experiments, both types of substrates were exposed to Ga flux alone during 10 seconds. The formation of Ga droplets was put in evidence by SEM (inset in figure 1a and 1b), illustrating the non-wettability of Ga on graphene. In a second set of experiments, graphene was exposed to both $\mathrm{Ga}$ and active $\mathrm{N}$ fluxes during about 15 minutes. The resulting nucleation seeds are shown in figure 1a (sample a) and $1 \mathrm{~b}$ (sample b). In both cases, the spatial distribution of $\mathrm{GaN}$ seeds reflects the Ga droplet distribution, as an evidence of the limited wettability of graphene by GaN. Furthermore, it clearly appears that seeds are decorating surface defects, namely the folds exhibited by $\mathrm{Gr} / \mathrm{SiO}_{2}$ and the steps characterizing $\mathrm{Gr} / \mathrm{SiC}$ surface. In addition, on $\mathrm{Gr} / \mathrm{SiO}_{2}$ a larger density of $\mathrm{GaN}$ seeds is observed on specific regions assigned to the presence of $2 \mathrm{LG}$ defects (see figure 1a). As shown in figure 1c and 1d, further growth of GaN leads to the formation of rough, imperfectly coalesced layers. 

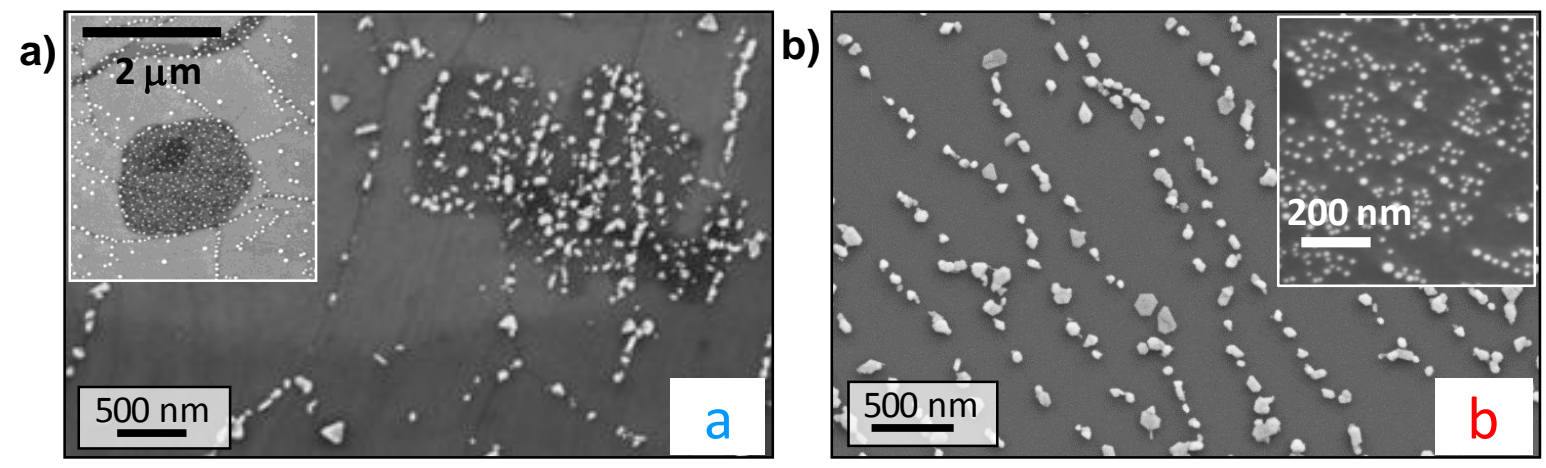

c)
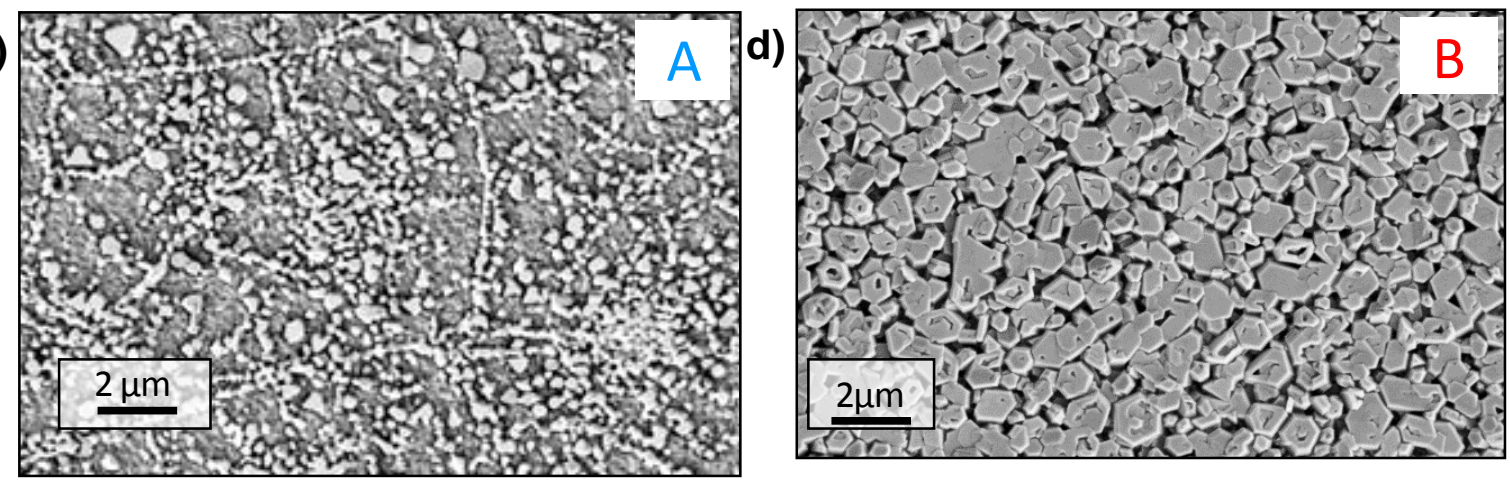

Figure 1. SEM images of a) sample $a, b)$ sample $b, c)$ sample A and d) sample B. Inset (a): $G a$ droplets resulting from exposure to $\mathrm{Ga}$ flux $\left(\mathrm{Gr} / \mathrm{SiO}_{2}\right.$ substrate). Dark areas correspond to $2 L G$ defects. Inset (b): Ga droplets resulting from exposure to Ga flux (Gr/SiC substrate).

The optical properties of both nucleation seeds and layers were characterized by photoluminescence (PL) spectroscopy performed at liquid helium temperature. As shown in figure 2, three main transitions are observed: a peak corresponding to the donor bound exciton $\left(\mathrm{D}^{0} \mathrm{X}\right)$ of wurtzite $(\mathrm{WZ}) \mathrm{GaN}(\sim 3.47 \mathrm{eV})$, one assigned to cubic zinc-blende (ZB) $\mathrm{GaN}$ at around $3.27 \mathrm{eV}$ and another one attributed to I1 type stacking faults (SFs) in GaN appearing within the $3.40-3.43 \mathrm{eV}$ range [21]. The relative intensity of these three peaks varies from sample to sample. Sample a is dominated by the $\mathrm{ZB} \mathrm{GaN} \mathrm{peak,} \mathrm{a} \mathrm{contribution} \mathrm{which} \mathrm{is} \mathrm{much}$ weaker for all the other samples, where we observe a high increase of the WZ GaN donor bound exciton transition. This feature suggests that c-oriented $\mathrm{WZ}$ grains are dominating and rapidly growing for thick samples (A and B) to the expense of randomly oriented ones and of ZB GaN. Remarkably, in figure $2 \mathrm{c}$, the FWHM of the $\mathrm{D}^{0} \mathrm{X}$ peak of sample $\mathrm{A}$ is as low as $6.9 \mathrm{meV}$ with 
a transition at $3.471 \mathrm{eV}$, assessing both the good structural quality and the relaxed state of individual grains in the layer, despite its rough morphology. One possible explanation for the larger FWHM of the $\mathrm{D}^{0} \mathrm{X}$ peak in sample $\mathrm{B}$ could be linked to the larger grain size leading to more strain inhomogeneity due to grain coalescence.

a)

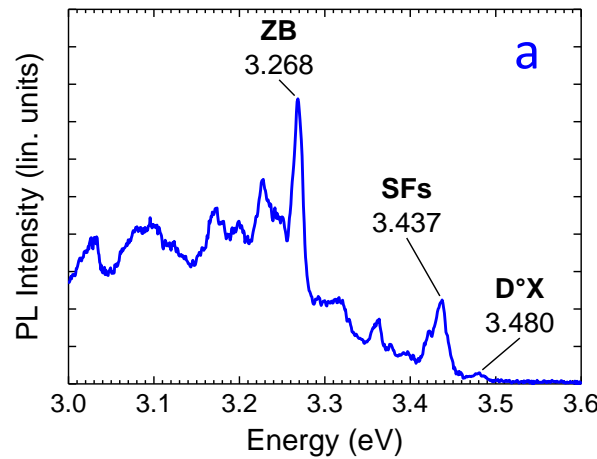

c)

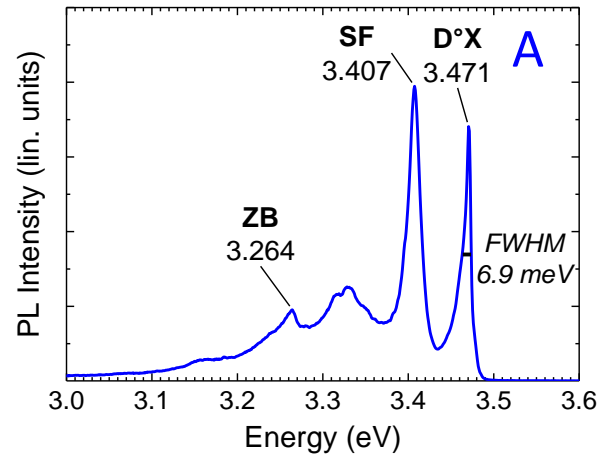

b)

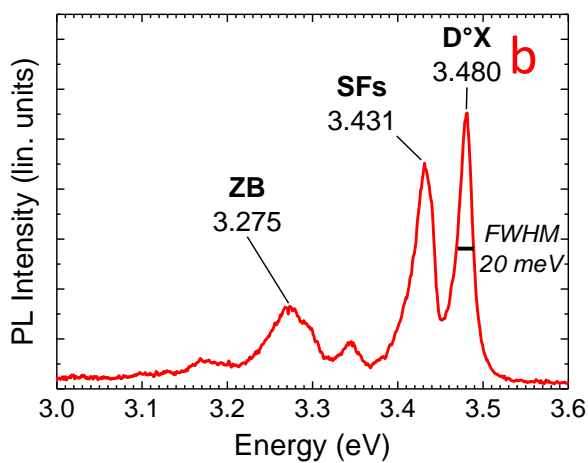

d)

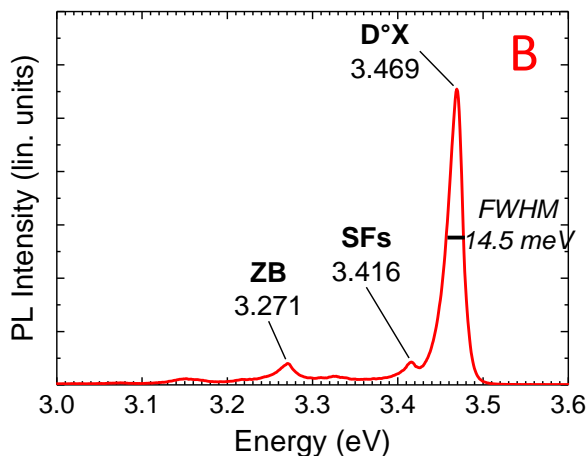

Figure 2: LT-PL of (a) sample $a$, (b) sample $b,(c)$ sample $A,(d)$ sample $B$.

The details of GaN nucleation at the nanoscale were investigated by performing high resolution transmission electron microscopy (HRTEM) experiments. The samples were prepared in cross-section by Focused Ion Beam (FIB). As shown in figure 3, corresponding to sample B, misoriented grains are visible in the very first stages of nucleation, with different orientations of their [00.1] direction with respect to the (00.1) $\mathrm{SiC}$ plane. As deduced from a Fourier transform and schematized in figure 3a, this includes grains exhibiting c-plane and mplane growth surface. Fully ZB grains were also identified, with their [111] axis either parallel 
to or tilted from the surface normal (see figure S1a in supplementary information). Above this perturbed region, WZ [00.1] GaN with a high density of stacking faults (SFs) is dominating, topped with unfaulted WZ [00.1] GaN. This piling sequence (see figure S1b in supplementary information) suggests that [00.1] oriented grains are growing to the expense of smaller, randomly nucleated grains on graphene, consistent with PL data. Figure $3 b$ shows a high-angle annular dark field (HAADF) image of the interfacial region between $\mathrm{GaN}$, graphene and SiC. The bright line visible above SiC corresponds to the intercalation of a 2 monolayer (ML) thick pure Ga layer under graphene. It was recently established that the formation of this metallic bilayer is mediated by metal diffusion from the defective graphene regions on $\mathrm{SiC}$ steps and further promoted during growth by the additional defects created by N-plasma in graphene [14]. In addition to the $\mathrm{Ga}$ bilayer, the presence of a $\mathrm{N}$ layer above $\mathrm{SiC}$ is revealed by energy dispersive Xrays (EDX) spectroscopy profiles taken along the sample normal (figure 3c). The presence of this layer is tentatively assigned to the presence of N-plasma-assisted defects in graphene, facilitating $\mathrm{N}$ diffusion below graphene. This process could be eased by the lack of coalescence of $\mathrm{GaN}$ islands shown in figure $1 \mathrm{~d}$ and limited to Ga-free regions on the surface which are directly exposed to $\mathrm{N}$ flux. However, due to the location of the $\mathrm{N}$ peak, coincident with the inflexion point of Si depth profile, it is also possible that diffusing $\mathrm{N}$ could be stabilized by forming N-Si bonds at the SiC surface. Although being desirable, a similar HRTEM study was not possible in the case of sample $\mathrm{A}$, due to the roughness of the $\mathrm{SiO}_{2}$ surface, which prevents from investigating the presence of metal and/or $\mathrm{N}$ layers intercalated in the case of $\mathrm{Gr} / \mathrm{SiO}_{2}$ 

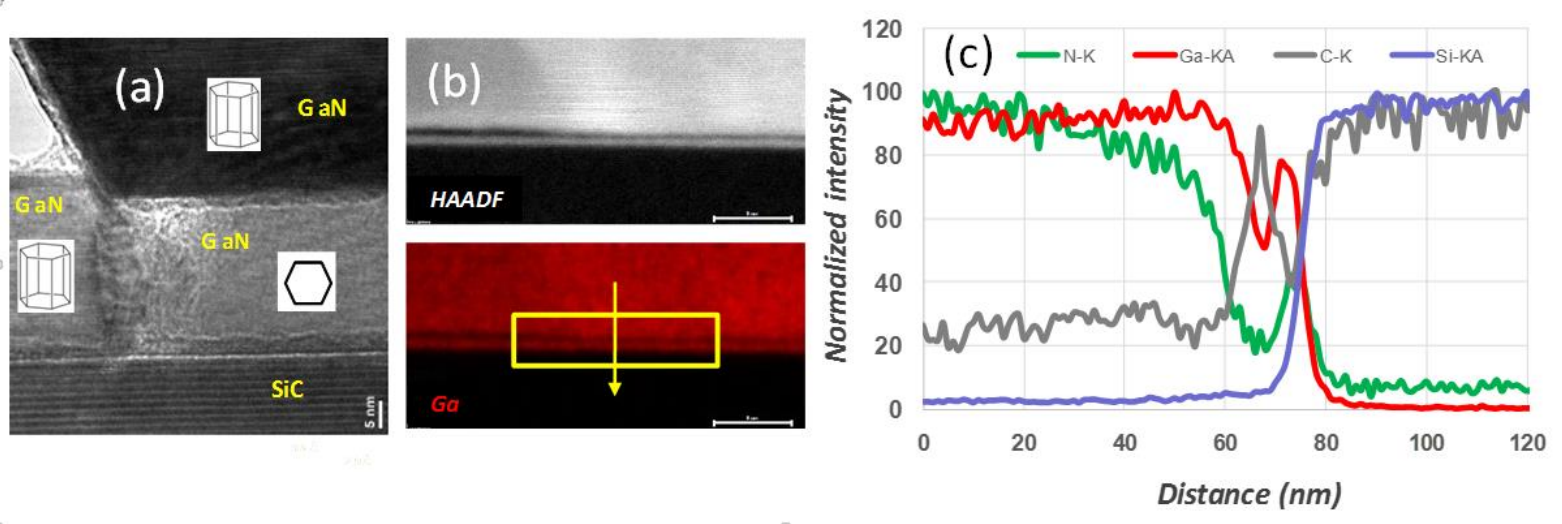

Figure 3: (a) HRTEM view of the SiC/Gr/GaN interface (sample B). The bright line above SiC corresponds to 2 MLs of Ga intercalated below graphene. The hexagonal prisms in inset indicate a c-axis perpendicular to the surface. A WZ grain with its c-axis lying parallel to the surface $\left(90^{\circ}\right.$-rotated hexagonal prism in inset) is visible, covered by WZ GaN with a large density of stacking faults b) STEM HAADF image of the interface region showing the Ga bilayer intercalated between SiC surface and graphene. c) EDX profile perpendicular to the surface, along the arrow shown in the integration window in b). Note the presence of Ga and $N$ layers below graphene.

The investigation of the GaN crystallographic phase mixing was extended to a larger scale, carrying out Raman spectroscopy experiments on GaN deposited on both types of substrates. Measurements were performed in backscattering configuration along the growth direction using the $532 \mathrm{~nm}$ line of a laser diode as excitation source. A Horiba Jobin Yvon Xplora spectrometer equipped with a Peltier-cooled charge coupled device detector and 1800 grooves/mm diffraction gratings was used for spectral analysis. A 100x microscope objective focused the excitation laser on the sample and collected the scattered light to the spectrometer. Figure 4 shows the Raman spectra of sample A and B measured in two different points of each sample (labeled A1, A2 and B1, B2, respectively). Three main peaks are present, assigned to the modes corresponding to the $\mathrm{WZ}$ crystal structure, $\mathrm{E}_{2 \mathrm{~h}}$ and $\mathrm{A}_{1}(\mathrm{LO})$, and to the $\mathrm{ZB}$ TO mode. 
The $\mathrm{E}_{2 \mathrm{~h}}$ peak is observed at $566.5 \mathrm{~cm}^{-1}$ in both samples, a frequency similar to that found in nanowires (NWs) and indicative of an almost strain-free material. The width of the $\mathrm{E}_{2 \mathrm{~h}}$ peak, which is a measure of crystal structure ordering, is very similar for both substrates, and amounts to $5 \mathrm{~cm}^{-1}$, somewhat larger than in typical HVPE free-standing samples $\left(3 \mathrm{~cm}^{-1}\right)$. In sample A, the $\mathrm{A}_{1}(\mathrm{LO})$ mode is observed at $735.5 \mathrm{~cm}^{-1}$, shifted by about $1.5 \mathrm{~cm}^{-1}$ from the expected 734 $\mathrm{cm}^{-1}$ value. This shift is assigned to phonon-plasmon coupling and is compatible with a $5 \times 10^{16}$ $\mathrm{cm}^{-3}$ free electron concentration. For the $\mathrm{Gr} / \mathrm{SiO}_{2}$ case, this peak is overdamped and cannot be detected, which indicates poor carrier mobility. An additional peak, ascribed to the ZB TO mode shown in spectra A1 and B1 appears at specific locations of the samples. This mode is narrower for $\mathrm{GaN}$ on $\mathrm{Gr} / \mathrm{SiO}_{2}$ than on $\mathrm{Gr} / \mathrm{SiC}\left(10 \mathrm{~cm}^{-1}\right.$ vs $\left.20 \mathrm{~cm}^{-1}\right)$, an effect assigned to the existence of domains of stacking faults and $\mathrm{ZB}$ material with larger size when $\mathrm{Gr} / \mathrm{SiO}_{2}$ is used as a substrate, resulting in a decrease in phonon disorder and a narrower peak. In both samples the peak assigned to the $\mathrm{ZB}$ phase is $3-4 \mathrm{~cm}^{-1}$ red-shifted with respect to the expected value $\left(555 \mathrm{~cm}^{-1}\right)$ [22], revealing a slight tensile strain due to the influence of the dominant WZ phase. Finally, it is important to stand out the clear absence in spectra B1 and B2 of the $\mathrm{A}_{1}(\mathrm{TO})$ mode, expected at $531 \mathrm{~cm}^{-1}$ (marked with an arrow), indicating that the GaN crystals are mainly oriented with the WZ $\mathrm{c}$ axis along the growth direction. This mode cannot be elucidated in spectra A1 and A2, since it is hidden by the large contribution from the silicon substrate. 


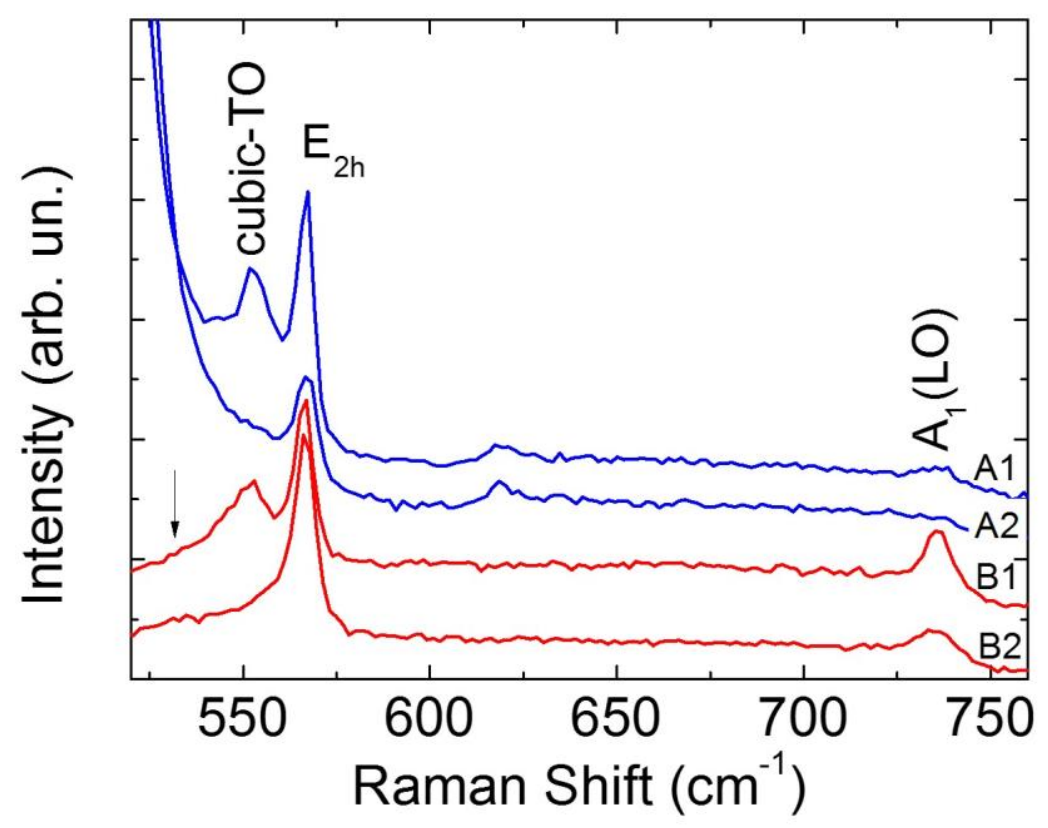

Figure 4: Raman spectra of samples $A$ and $B$. Two spectra for each (A1, A2, B1, B2) correspond to two different regions in the samples. Besides the modes corresponding to the WZ crystal structure [ $E_{2 h}$ and $\left.A_{l}(L O)\right]$, spectra $A 1$ and $B 1$ also show contributions from $Z B$ GaN at specific locations of the samples. The arrow shows the expected frequency of $A_{1}(T O)$ mode.

In order to evaluate the local distribution of $\mathrm{ZB}$ and $\mathrm{WZ}$ phase, Raman maps were performed by scanning a $10 \times 10 \mu \mathrm{m}^{2}$ area with a $1 \mu \mathrm{m}$ step. The result for sample B is plotted in figure $5 \mathrm{a}, 5 \mathrm{~b}$ and $5 \mathrm{c}$ superposed to the corresponding optical micrograph. The figures show intensity maps of the ZB TO mode, the $\mathrm{E}_{2 \mathrm{~h}}$ mode, and the $\mathrm{A}_{1}(\mathrm{LO})$, respectively. The $\mathrm{E}_{2 \mathrm{~h}}$ and $\mathrm{A}_{1}(\mathrm{LO})$ modes are present in all spectra with similar frequency and width, but marked intensity fluctuations. These variations are emphasized in figure $5 \mathrm{~b}$ and $5 \mathrm{c}$ by representing only intensities larger than 1300 and 550 arbitrary units, respectively. The strong spatial correlation of the intensity of both modes reflects fluctuations in the GaN coverage. On the contrary, the cubic mode is clearly absent from most of the studied regions. It also exhibits strong intensity 
fluctuations, but not directly correlated with those of the WZ material. Similar results were obtained in the case of sample A (see supplementary information), suggesting that crystallographic phase mixing is inherent to $\mathrm{GaN}$ growth on graphene, either transferred on $\mathrm{SiO}_{2}$ or grown on $\mathrm{SiC}$. As pointed out before, the $\mathrm{A}_{1}(\mathrm{TO})$ mode, forbidden in backscattering geometry along the $\mathrm{WZ} \mathrm{c}$ axis, is absent in all the spectra. This is a clear indication that $\mathrm{GaN}$ grains with their c-axis perpendicular to the surface prevail.

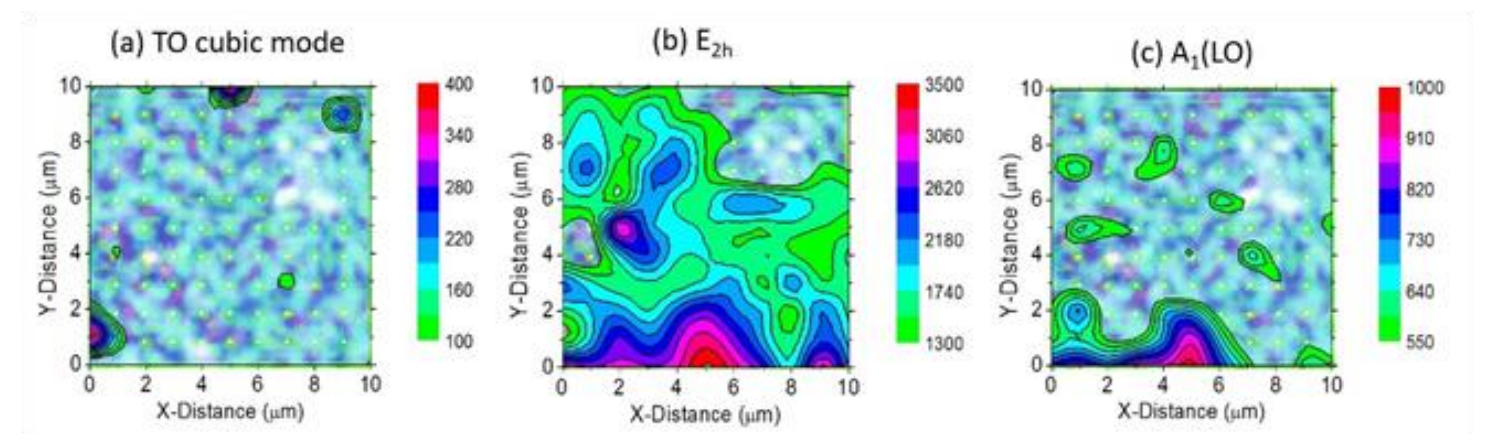

Figure 5: Raman maps of sample B superposed to the associated optical microscope image. a) ZB TO mode, b) $E_{2 h}$ mode, c) $A_{l}(L O)$ mode

Both samples present, however, important differences concerning the characteristics of the graphene substrate after GaN growth. As shown in the supplementary information (figure S2 d) the 2D mode of graphene is absent from almost all the investigated points of sample A, indicating a severe damage of the graphene layer after GaN growth. On the contrary, for sample $\mathrm{B}$, the $2 \mathrm{D}$ mode is present in all the points, although with significant intensity variations (figure $\mathrm{S} 3 \mathrm{c}$ ). This result points out that, during $\mathrm{GaN}$ growth, graphene/SiC is better preserved from damage than graphene/ $\mathrm{SiO}_{2}$.

It is worth stressing that $\mathrm{GaN}$ deposited directly on $6 \mathrm{H}-\mathrm{SiC}$ grows in a simple epitaxial relationship, with [00.1] $\mathrm{GaN} / /[00.1] \mathrm{SiC}$ and [1-1.0] $\mathrm{GaN} / /[1-1.0] \mathrm{SiC}$ [23, 24]. By contrast, the present observation of almost relaxed, misoriented $\mathrm{GaN}$ grains on $\mathrm{Gr} / \mathrm{SiC}$ is assigned to the loss of interfacial strength bonding and to the weak interaction of epitaxial GaN with the 
substrate, a feature inherent to the nature of $\mathrm{VdW}$ epitaxy. In other words, it is expected that the weak interaction between the epilayer and the VdW substrate leads to the breaking of the conventional epitaxial rules observed in the case of three-dimensional epitaxy.

To test this hypothesis, X-Rays diffraction (XRD) experiments were performed using a Rigaku SmartLab X-Ray diffractometer system in standard $2 \theta-\theta$ and in grazing incidence configurations in order to assess the out-of-plane and the in-plane orientations of the $\mathrm{GaN}$ layers. In addition to samples A and B, we also measured samples NW1 and NW2 to investigate the role of $\mathrm{Ga} / \mathrm{N}$ flux ratio in the nucleation process on graphene, whatever the sub-substrate. 2 $\theta-\theta$ XRD out-of-plane scans shown in figure 6 are dominated by the $(00.2 \mathrm{n})$ peaks corresponding to c-oriented $\mathrm{WZ}$ GaN. Besides these peaks additional peaks assigned to misoriented grains are visible. More precisely, peaks corresponding to grains with (10.0), (10.1), (11.0), (10.3) (11.2) and (30.0) orientations as well as peaks corresponding to the GaN ZB phase are identified for both A and B samples, as an evidence that the nature of the subjacent substrate below graphene is not determinant, namely $\mathrm{SiC}$ or $\mathrm{SiO}_{2}$. For sample NW2 some of these additional peaks, less intense, are also seen in the diffractogram. However, in the case of sample NW1 the diffractogram only shows WZ with c-axis perpendicular to the surface, demonstrating that in this case NW nucleation is widely dominated by seeds with WZ c-axis perpendicular to the surface.

This feature is consistent with previous reports on the growth of c- oriented GaN NWs on graphene on $\mathrm{SiO}_{2}$ [8] and graphene on $\mathrm{SiC}$ [9], with no evidence of $\mathrm{ZB}$ grains or $\mathrm{SFs}$ at the GaN/graphene interface. It confirms that the chemical nature of the subjacent substrate below graphene does not significantly affect NW growth. It also demonstrates that the detailed growth conditions, in particular the $\mathrm{Ga} / \mathrm{N}$ flux ratio value, are controlling nucleation and that misoriented seeds are correlated with a $\mathrm{Ga} / \mathrm{N}$ flux ratio value higher than 1 . 


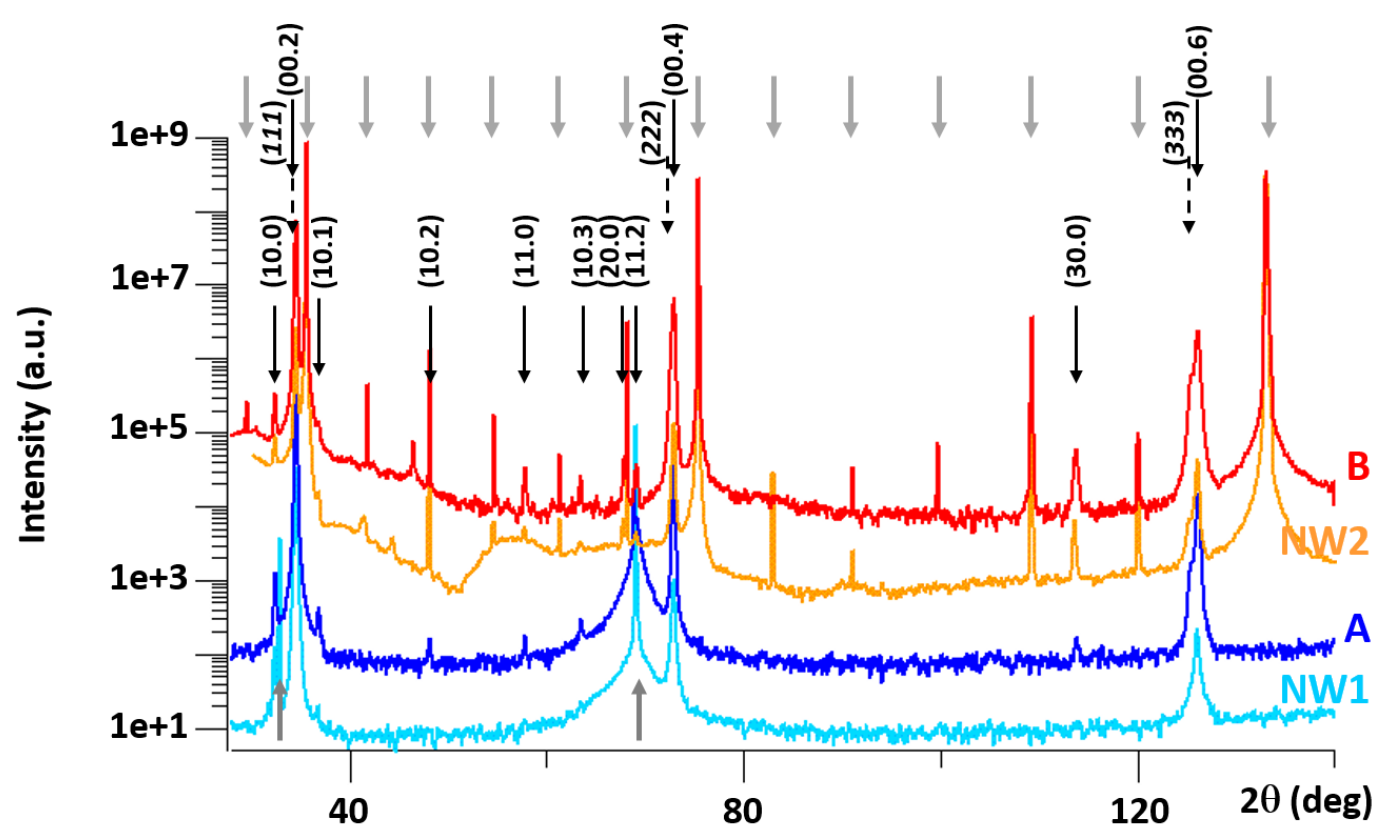

Figure 6: $2 \theta-\theta$ scan of samples NW1, A, NW2 and B. The grey arrows on the top refer to the SiC substrate reflections (00.n) while the grey arrows on the bottom to the Si substrate reflections (00n) under the $\mathrm{SiO}_{2}$ layer. The (hkl) reflections in italics are from the $\mathrm{ZB} \mathrm{GaN}$ phase. All the others reflections are indexed in the WZ phase (black arrows). The curves were shifted for the sake of clarity.

We speculate that the weak VdW interactions between epilayer and substrate may permit the nucleation of metastable GaN orientations not found in traditional 3D epitaxy. With the purpose of quantitatively supporting the above statements, discreet calculations of lattice site coincidence for various growth orientations of $\mathrm{GaN}$ on graphene were performed. Only the $\mathrm{GaN}$ epilayer and graphene subjacent substrate were considered in this calculation. It was assumed that $\mathrm{Ga}$ atoms adsorb on exclusively H6 lattice sites at the center of carbon rings as theoretically proposed by Nakada et al [25]. These sites (as opposed to sites occupied by carbon atoms) form their own sublattice, on top of which Ga atoms are considered to be at a minimum separation. The impact of a VdW gap was accounted for by employing a Lennard-Jones potential to approximate the relative interaction strength of $\mathrm{Ga}$ atoms and adsorption (H6) sites 
[26]. In the equation for this potential, for simplicity, $\varepsilon$ (potential well depth) was taken to be 1 and the atom separation at minimum potential energy $\left(r_{m}\right)$ was set as the VdW gap. The separation between each Ga atom and nearest nucleation site (H6 site) is given by " $r$ ". Chemical species arrangement within the GaN WZ lattice cell was disregarded and only a single species was included in each calculation (in this case $\mathrm{Ga}$, though $\mathrm{N}$ could equally have been selected due to its identical sublattice). All results are presented as the potential energy per Ga atom at the interface:

$$
V_{L J}=\varepsilon\left[\left(\frac{r_{m}}{r}\right)^{12}-2\left(\frac{r_{m}}{r}\right)^{6}\right]
$$

A nearest neighbour approximation was employed. However when considering a large VdW gap relative to the interatomic spacing of the Ga atoms this approximation may be insufficient.In practice values of the $\mathrm{VdW}$ gap ranging from 0.001 to $1 \mathrm{~nm}$ were considered in the calculations. Moreover, it is worth recalling that the LJ potential models the interaction strength of isolated neutral atoms. This is expected to be a reasonable approximation due to the lack of covalent bonding in VdW epitaxy, nonetheless the comparison between different local interaction energy minima should require a more complete model accounting for different chemical species, namely $\mathrm{N}$ and $\mathrm{Ga}$, and their ionic nature. Additionally the presence of a bilayer of graphene and $\mathrm{Ga}$ intercalated layer is expected to limit any memory of the SiC template.

By rotation of GaN grains around two principal axes we searched for non c-plane orientations of $\mathrm{GaN}$ on graphene that yield strong lattice coincidence. Minima in these plots indicate preferred epitaxial orientations. The two rotations considered were (1) rotation of GaN around the [10.0] direction with the GaN/graphene interface varying from the $\mathrm{c}$ to the m-plane, and (2) a $30^{\circ}$ rotation of $\mathrm{GaN}$ around the [00.1] followed by rotation around its [11.0] direction, where the interface varies from the $\mathrm{c}$ to the a-plane. A Ga array consisting of $\sim 150$ hexagonal 
cells was employed to compensate for the periodic artefacts induced when a new face is generated through an arbitrary termination of the lattice.

The results of the calculations assuming an $r_{m}$ value of $0.001 \mathrm{~nm}$, i.e. neglecting the VdW gap, are shown in figure 7. As expected, one minimum corresponds to c-plane $\mathrm{GaN}(\alpha=$ $\left.0^{\circ}\right)$ i.e. to the dominant $(00.1)$ oriented $\mathrm{WZ} \mathrm{GaN} \mathrm{islands} \mathrm{measured} \mathrm{in} \mathrm{TEM} \mathrm{and} \mathrm{X-Ray} \mathrm{data.}$ Minima also occur for $\mathrm{m}$ and a-plane orientations $\left(\alpha=90^{\circ}\right)$, both of which are present in the XRD data as (10.0), (20.0), (30.0) and (11.0) reflections. Other local minima correspond to the (10.1), (20.1) and (30.1) orientations at $\alpha=32^{\circ}, 51^{\circ}$ and $62^{\circ}$ respectively while only the most pronounced (10.1) is observed in the XRD data.

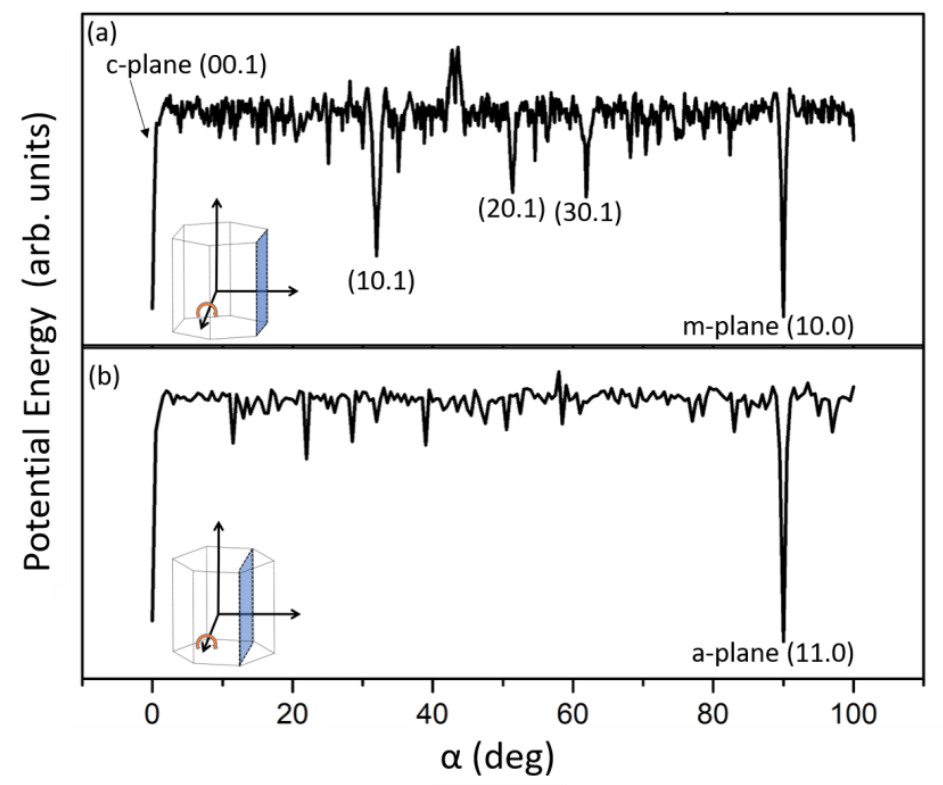

Figure 7: Per-atom potential energy of GaN on graphene for rotation $(\alpha)$ about the (a) [10.0] and (b) [11.0] directions for $r_{m}=0.001 \mathrm{~nm}$. Local minima occur for each principal direction as well as at $32^{\circ}(10.1), 51^{\circ}(20.1)$ and $62^{\circ}(30.1)$ tilt in the direction of the m-plane. The principal

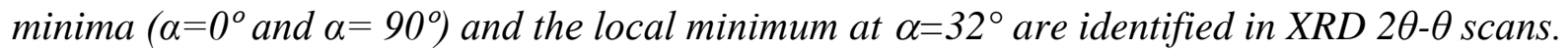


In a further set of X-ray experiments, we analysed the in-plane epitaxial relation with graphene for the dominant (00.1) oriented WZ GaN islands. For this purpose, grazing incidence $\phi$-scans were performed at the (11.0) $\mathrm{GaN}$ reflection. For the growth of $\mathrm{GaN}$ on $\mathrm{Gr} / \mathrm{SiO}_{2}$, as the graphene is polycrystalline, no preferential orientation could be clearly identified. The results for growth on $\mathrm{Gr} / \mathrm{SiC}$ are reported in figure 8 , where the reference position $(\phi=0)$ corresponds to the [11.0] $\mathrm{SiC}$ direction. For the 3 samples the background intensity is much higher than the background intensity of the experimental setup (see background level of SiC). The effect is even stronger for sample a (not shown). This indicates that the nucleation on graphene is totally misoriented. As expected, for samples B and NW2 the main in-plane orientation of $\mathrm{GaN}$ is observed at $0^{\circ}$, i.e. $30^{\circ}$-rotated with respect to graphene, which is itself $30^{\circ}$-rotated with respect to subjacent $\mathrm{SiC}$ [27].

Remarkably, several additional epitaxial relations are found for the nucleation layer (sample b), at $11^{\circ}, 27^{\circ}$ and $30^{\circ}$ (modulo 60 degrees due to the 6-fold symmetry of the WZ structure around the $\mathrm{c}$ axis), each one exhibiting a different width and different intensity. $2 \theta-\phi$ scans were performed for every angle of rotation (data not shown) allowing one to discard the hypothesis of a size effect and assess the peak width to in-plane twist. As shown later, calculations have established that their position and relative intensity drastically depends on the value of the VdW gap. However, a quantitative fit of the VdW gap would require using a more complete potential to account for the details of atomic interaction. 


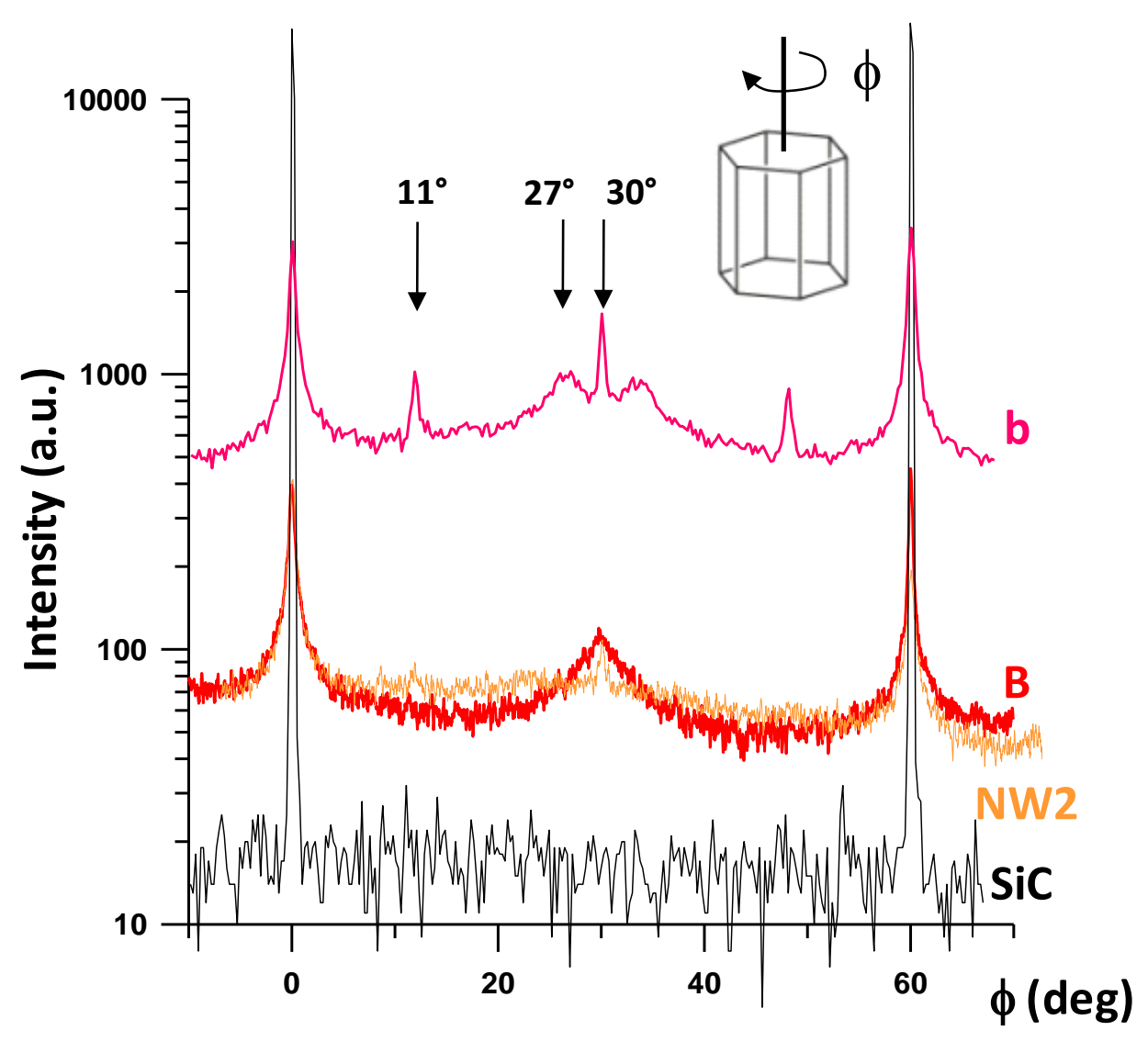

Figure 8: In plane grazing incidence $\phi$-scan around the (11.0) GaN reflection for sample a, A and NW1. As a reference the scan for the (11.0) reflection of SiC is also shown. Note the presence of several peaks at $11^{\circ}, 27^{\circ}$ and $30^{\circ}$ from the SiC reference. No shift in intensity was applied.

In order to obtain the preferred in-plane epitaxial relations (preserving [00.1] as the growth direction), a similar procedure to that outlined above was employed but taking into account only a single $\mathrm{GaN}$ hexagon ( $7 \mathrm{Ga}$ atoms, the central $\mathrm{Ga}$ atom and the 6 surrounding ones) rotated around its [00.1] direction. Again, the Ga atom was allowed to interact only with the H6 sub-lattice. The VdW gap for this calculation was initially set to $0.001 \mathrm{~nm}$ so as to be negligible and subsequently increased to investigate its impact on the number and positions of potential minima. These occur at rotations of maximal overlap between Ga and H6 lattice sites. As expected from the XRD data the calculated minima correspond to the observed preferred 
orientation, with the [11.0] direction of the GaN lattice parallel to the [10.0] direction of graphene, also referred to as carbon zigzag chains [8] (see inset in figure 9, with Ga assumed to be adsorbed on an $\mathrm{H} 6$ site). They repeat with a $60^{\circ}$ periodicity owing to the symmetry of $\mathrm{WZ}$ GaN. Increasing the separation (VdW gap) between film and substrate till a value of $0.3 \mathrm{~nm}$ consistent with experimental determination [14] leads to a broadening of the potential energy dispersion.

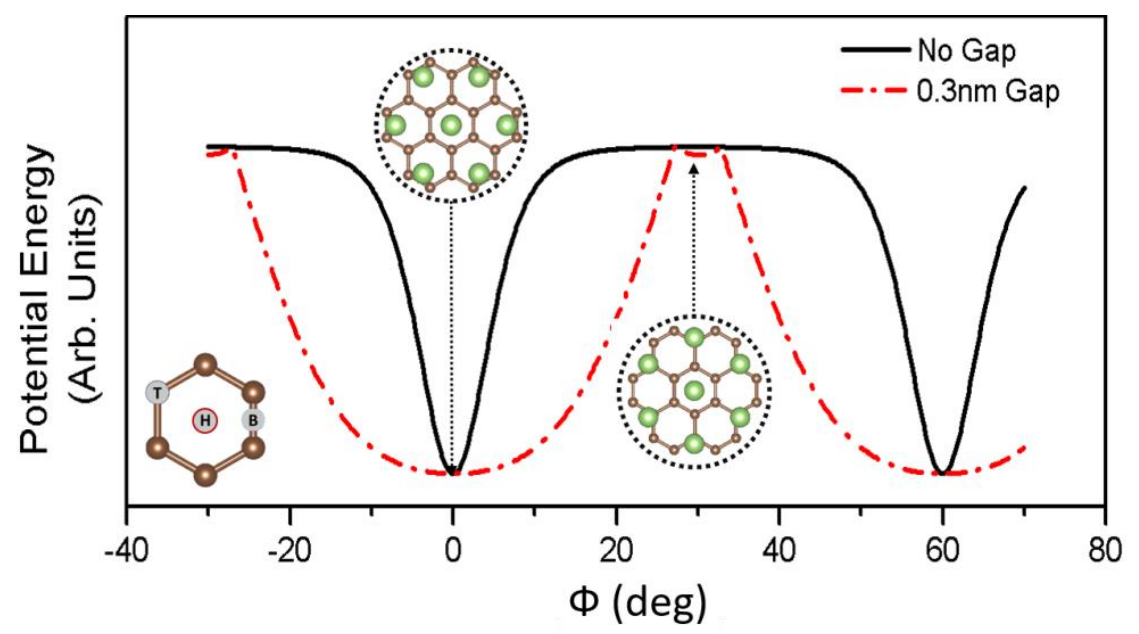

Figure 9: Per-atom LJ potential energy of a single Ga sublattice hexagonal cell as it is rotated around the [00.1] direction on a fixed graphene substrate. According to the scheme in inset, Ga is adsorbed on the H site. $0^{\circ}$ and $60^{\circ}$ correspond to the GaN [10.0] direction parallel to the graphene [11.0] direction which is the preferred orientation [8]. The position of the Ga atoms with respect to the graphene lattice is shown schematically in the circled insets where Ga atoms are in green and $C$ atoms in brown for $0^{\circ}$ and $30^{\circ}$. Increasing the size of the VdW gap to 0.3 $n m$ (red) results in a broadening of the energy dispersion near the minima.

As previously noted, additional local energy minima are found from the preferred epitaxial relationship in XRD data shown in figure 8. Intermediate orientations were previously reported at $19^{\circ}$ correlated to the existence of preferential $\mathrm{Ga} / \mathrm{N}$ adsorption sites on the graphene surface at the midpoint between graphene atoms (B sites) [25, 28]. Indeed, when the discreet 
calculation is repeated for exclusively B-sites (B-site sublattice) without considering the VdW gap, new minima are observed at $\phi=19^{\circ}$ and $41^{\circ} \quad$ (figure 10).

However, by increasing the size of the VdW gap while allowing Ga to interact with the B-site sublattice, a central $0^{0}$ minimum is restored with several surrounding "satellites" (figure 10). Asymmetric broad minima can also be observed to emerge near $28^{\circ}$ and $32^{\circ}$. In particular, assuming a $\mathrm{VdW}$ gap of $0.3 \mathrm{~nm}$, minima are found at $12^{\circ}, 28^{\circ}$ and $32^{\circ}$. These simulations are then in semi-qualitative agreement with XRD data (excepting for the lack of a $30^{\circ}$ minimum), suggesting that the satellites are related to the occupation of both $\mathrm{H} 6$ and B adsorption sites on graphene and drastically dependent on the size of the VdW gap. The correspondence between our model and experimental results furthermore suggests epitaxial registry is dominant over other possible contributions to nucleation including structural defects in the graphene induced by $\mathrm{N}$ plasma. Further improvement of the calculations to quantitatively account for the positions of satellites will require taking into account the full chemical structure of GaN, which consists of chemically bonded $\mathrm{N}$ and Ga hexagonal lattices, as well as optimizing the form of the potential. We also speculate that the satellites may be attributed to a superposition of sublattices not considered, such as the combined H6+B lattice, whose probability of occupation depend on the chemical nature of the atoms, i.e. $\mathrm{N}$ or $\mathrm{Ga}$.

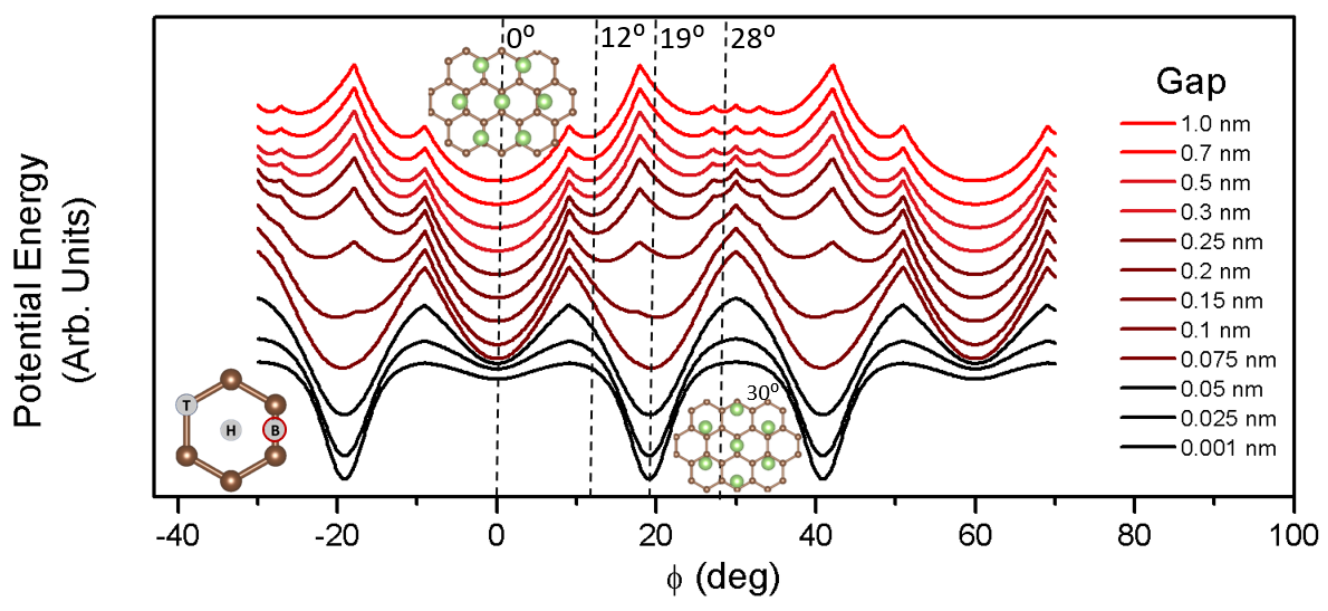


Figure 10: Per-atom potential energy of a single hexagonal sublattice cell as it is rotated around (00.1) on the graphene B-site sublattice (after [25]) (see the bottom left inset, with $C$ atoms in brown and Ga atoms in green). For the "No Gap" calculation, the minima are at $19^{\circ}$ and $41^{\circ}$. When a gap is introduced, the $0^{\circ}$ minimum is recovered with additional local minima satellites. The position of the Ga atoms with respect to the graphene lattice is also depicted schematically at their respective $0^{\circ}$ and $30^{\circ}$ positions, where Ga atoms are in green and $C$ atoms in brown.

In summary, both $2 \theta-\theta$ and (11.0) $\mathrm{GaN} \phi$-scans have revealed that the epitaxial relationships between $\mathrm{GaN}$ and either $\mathrm{Gr} / \mathrm{SiO}_{2}$ or $\mathrm{Gr} / \mathrm{SiC}$ are complex, with a variety of $\mathrm{ZB}$ and WZ GaN misoriented grains on the one hand, and twisted (00.1) oriented grains on the other hand. Furthermore, the degree of misorientation was found greater in the thick layer compared to NWs, as a clue that detailed growth conditions play a major role.

More precisely, the above results point out the role of $\mathrm{Ga} /$ active nitrogen flux ratio value on the GaN nucleation process on graphene. Whereas initiating the growth in N-rich conditions results in the formation of $c$-oriented WZ NWs $[8,9]$, the present study has shown that nucleation in Ga-rich conditions is associated with the formation of a mixture of randomly oriented grains and of $c$-oriented ones, followed by progressive predominance of the latter for increasing $\mathrm{GaN}$ layer thickness.

Due to the presence of a nucleation barrier, it has been established that GaN heteroepitaxial nucleation is nonlinear with time, which is assigned to a reduced Ga sticking coefficient in the early stages of growth [29]. In the case of GaN NWs, this leads to the occurrence of a so-called incubation time, prior to nuclei formation. The duration of the incubation time is directly linked to the Ga adatom density on the surface, which itself depends on growth temperature and Ga flux values [30]. Consequently, GaN nucleation on graphene is 
expected to drastically depend on the value of $\mathrm{Ga}$ active $\mathrm{N}$ flux ratio. High values associated with large nuclei density and limited or no incubation time are expected to potentially result in nucleation in all the favorable points in the GaN/graphene coincidence lattice. By contrast, in the case of low values of the $\mathrm{Ga}$ /active $\mathrm{N}$ flux ratio typically corresponding to $\mathrm{NW}$ growth conditions, the long incubation time leads to preferential nucleation on the most stable points of the coincidence lattice, to the expense of metastable ones. In the absence of a strong relationship to the substrate, the shape of the nuclei is expected to be governed by thermodynamics. As a matter of fact, the stability shape of GaN has been examined by Hong Li et al [31]. In particular, a c-oriented rod-like structure was found to be the GaN equilibrium shape, consistent with the observations on the growth of GaN NWs on a variety of substrates including amorphous $\mathrm{SiO}_{2}[32]$.

Whereas the experimentally observed epitaxial relationships are qualitatively predicted by the lattice coincidence model, it fails to fully account for the position of the satellites XRD peaks around the preferred epitaxial orientation. As discussed above, improvement of the model would require an optimization of the interaction potential and taking into account the different chemical nature of both types of atoms involved, namely $\mathrm{N}$ and $\mathrm{Ga}$. However it is also likely that N-plasma-induced defects could significantly influence the GaN nucleation process. Indeed, exposure of graphene to N-plasma results in the formation of a variety of defects. These include the formation of out-of-plane $\mathrm{C}-\mathrm{N}$ bonds which could contribute to the formation of covalent bonding between $\mathrm{N}$-doped graphene and GaN layer [33, 34]. Consistently, Raman experiments reported here have put in evidence drastic modifications of graphene either transferred on $\mathrm{SiO}_{2}$ or grown on $\mathrm{SiC}$, assigned to the exposure to $\mathrm{N}$ plasma.

\section{Conclusion}


In conclusion, it has been shown that conventional epitaxial rules observed in the case of three-dimensional epitaxy do not hold in the case of GaN thick layers grown by MBE on graphene. It has been furthermore demonstrated that the nature of the supporting substrate is not relevant and that kinetically-driven polycrystalline nucleation is dominant, pointing out the details of the growth conditions, namely temperature and $\mathrm{Ga}$ active $\mathrm{N}$ flux ratio value. The role of GaN/graphene coincidence lattice on the orientation of individual grains has been emphasized. Whereas random seed orientation was obtained for high $\mathrm{Ga} /$ active $\mathrm{N}$ flux ratio, single $c$-oriented seed orientation could be obtained for low $\mathrm{Ga} /$ active $\mathrm{N}$ flux ratio corresponding to NW growth conditions. The $500 \mathrm{~nm}$ thick layers are strain-relaxed and exhibit a structural quality comparable to $\mathrm{GaN}$ thick layers grown on classical substrates as attested by photoluminescence, Raman spectroscopy and XRD analysis. However, the lack of coalescence of these layers points out the lack of wetting intrinsically associated with the weak interaction, characteristic of $\mathrm{VdW}$ epitaxy, making graphene a substrate particularly adapted to the growth of nanowires while the growth of continuous layers on a large surface is expected to be challenging.

\section{Acknowlegments}

The authors are grateful to Timotée Journot and Bérangère Hyot for providing the graphene/ SiC substrates and acknowledge N. Mollard for the preparation of TEM samples. AC and NG acknowledge financial support from project ENE2016-79282-C5-3-R cofinanced by the Spanish MICINN and FEDER funds and PROMETEO/2018/123 from Generalitat Valenciana.

\section{Author contributions}


BD and MG initialize the idea. AC and NG designed Raman spectroscopy experiments and analyzed the data. MG and NF have grown and characterized the samples. MG, NF and BG performed the photoluminescence spectroscopy experiments and analyzed the data. NF made the theoretical calculations. EBA and SP performed XRD experiments. CB and HO performed high resolution electron microscopy and EDX experiments. All authors discussed the results and contributed to the manuscript.

\section{Competing interests}

The authors declare no competing interests. 


\section{References}

[1] Koma A 1992 Van der Waals epitaxy - a new epitaxial growth method for a highly latticemismatched system Thin Solid Films 216 72-6

[2] Walsh L A, Hinkle C L 2017 van der Waals epitaxy: 2D materials and topological insulators Applied Materials Today 9 504-15

[3] Xenogiannopoulou E, Tsipas P, Aretouli K E, Tsoutsou D, Giamini S A, Bazioti C, Dimitrakopulos G P, Komninou Ph, Brems S, Huyghebaert C, Raduc I P and Dimoulas A 2015 High-quality, large-area $\mathrm{MoSe}_{2}$ and $\mathrm{MoSe}_{2} / \mathrm{Bi}_{2} \mathrm{Se}_{3}$ heterostructures on AlN(0001)/Si(111) substrates by molecular beam epitaxy Nanoscale 7 7896-7905

[4] Xia C, Johansson L I, Zakharov A A, Hultman L and Virojanadara C 2014 Effects of Al on epitaxial graphene grown on 6H-SiC(0001) Materials Research Express 1015606

[5] Xu Y, Cao B, Li Z, Cai D, Zhang Y, Ren G, Wang J, Shi L, Wang C and Xu K 2017 Growth Model of van der Waals Epitaxy of Films: A Case of AlN Films on Multilayer Graphene/SiC ACS Applied Material Interfaces 9 44001-9

[6] Araki T, Uchimura S, Sakaguchi J, Nanishi Y, Fujishima T, Hsu A, Kim K K, Palacios T, Pesquera A, Centeno A and Zurutuza A 2014 Radio-frequency plasma-excited molecular beam epitaxy growth of $\mathrm{GaN}$ on graphene/Si(100) substrates Applied Physics Express 7 071001-3

[7] Kim J, Bayram C, Park H, Cheng C-W, Dimitrakopoulos C, Ott J A, Reuter K B, Bedell S W and Sadana D K 2014 Principle of direct van der Waals epitaxy of single-crystalline films on epitaxial graphene Nature Communications 55836

[8] Kumaresan V, Largeau L, Madouri A, Glas F, Zhang H, Oehler F, Cavanna A, Babichev A, Travers L, Gogneau N, Tchernycheva M and Harmand J-C 2016 Epitaxy of GaN Nanowires on Graphene Nano Letters 16 4895-4902

[9] Fernández-Garrido S, Ramsteiner M, Gao G, Galves L A, Sharma B, Corfdir P, Calabrese G, de Souza Schiaber Z, Pfüller C, Trampert A, Marcelo J. Lopes J, Brandt O and Geelhaar L 
2017 Molecular Beam Epitaxy of GaN Nanowires on Epitaxial Graphene Nano Letters 17 $5213-5221$

[10] Journot T Bouchiat V, Gayral B, Dijon J and Hyot B 2018 Self-Assembled UV Photodetector Made by Direct Epitaxial GaN Growth on Graphene ACS Appl. Mater. Interfaces $1018857-18862$

[11] Mun D-H Bae H, Bae S, Lee H, Ha J-S, and Lee S 2014 Stress relaxation of GaN microstructures on a graphene-buffered $\mathrm{Al}_{2} \mathrm{O}_{3}$ substrate Phys. Status Solidi RRL 8 341-344

[12] Li Y, Zhao Y, Wei T, Liu Z, Duan R, Wang Y, Zhang X, Wu Q, Yan J, Yi X, Yuan G, Wang J and Li J 2017 Van der Waals epitaxy of GaN-based light-emitting diodes on wettransferred multilayer graphene film Japanese Journal of Applied Physics 56085506 1-6

[13] Al Balushi Z Y, Miyagi T, Lin Y-C, Wang K, Calderin L,Bhimanapati G, Redwing J M, and Robinson J A 2015 The impact of graphene properties on GaN and AlN nucleation Surface Science 634 81-88

[14] Feldberg N, Klymov O, Garro N, Cros A, Mollard N, Okuno H, Gruart G and Daudin B 2019 Spontaneous intercalation of Ga and In bilayers during plasma-assisted molecular beam epitaxy growth of GaN on graphene on SiC Nanotechnology 30375602 1-9

[15] Sun X, Lu Z, Xie W, Wang Y, Shi J, Zhang S, Washington M A and Lu T M 2017 van der Waals epitaxy of CdS thin films on single-crystalline graphene Applied Physics Letters 110 $1531041-5$

[16] Boschker J E, Galves L A, Timur Flissikowski T, Lopes J M J, Riechert H and Calarco R 2015 Coincident-site lattice matching during van der Waals epitaxy Scientific Reports 518079

[17] Boschker J E, Momand J, Bragaglia V, Wang R, Perumal K, Giussani A, Bart J. Kooi B J, Riechert H and Calarco R 2014 Surface Reconstruction-Induced Coincidence Lattice Formation Between Two-Dimensionally Bonded Materials and a Three-Dimensionally Bonded Substrate Nano Letters 14 3534-3538

[18] https://www.graphenea.com/ 
[19] Michon A, Largeau L, Mauguin O, Ouerghi A, Vézian S, Lefebvre D, Roudon E, Zielinski M, Chassagne T, and Portail M 2012 Graphene growth using propanehydrogen CVD on 6H-SiC(0001): temperature dependent interface and strain Physica Status Solidi C 9 $175-178$

[20] Michon A, Vézian S, Roudon E, Lefebvre D, Zielinski M, Chassagne T, and Portail M 2013 Effects of pressure, temperature, and hydrogen during graphene growth on $\mathrm{SiC}(0001)$ using propane-hydrogen chemical vapor deposition Journal of Applied Physics 113, 203501 18

[21] Liu R, Bell A, Ponce F A, Chen C Q, Yang J W, and Khan M A 2005 Luminescence from stacking faults in gallium nitride Applied Physics Letters 86, 021908 1-3

[22] Siegle H, Eckey L, Hoffmann A, Thomsen C, Meyer B K, Schikora D, Hankeln M, and Lischka K 1995 Quantitative determination of hexagonal minority phase in cubic GaN using Raman spectroscopy Solid State Communications 96 943-949

[23] Liu L and Edgar J H 2002 Substrates for gallium nitride epitaxy Materials Science and Engineering $R 37$ 61-127

[24] Rauschenbach B, Lotnyk A, Neumann L, Poppitz D and Gerlach J W 2017 Ion Beam Assisted Deposition of Thin Epitaxial GaN Films Materials 10690

[25] Nakada K and Ishii A 2011 Migration of adatom adsorption on graphene using DFT calculation Solid State Communications 151 13-16

[26] Lennard-Jones J E 1924 On the Determination of Molecular Fields - II. From the equation of state of a gas Proceedings of Royal Society London A, 106, 463

[27] Adrien Michon A, Ludovic Largeau L, Antoine Tiberj A, Jean-Roch Huntzinger J R, Olivia Mauguin O, Stéphane Vézian S, Denis Lefebvre D, Fabien Cheynis F, Frédéric Leroy F, Pierre Müller P, Thierry Chassagne T, Marcin Zielinski M, and Marc Portail M 2013 $\mathrm{X}$-ray diffraction and Raman spectroscopy study of strain in graphene films grown on $6 \mathrm{H}-$ SiC(0001) using propane-hydrogen-argon CVD Materials Science Forum 740-742 117-120 
[28] Sarau G, Heilmann M, Latzel M, Tessarek C and Christiansen S 2019 GaN-Based Nanorods/Graphene Heterostructures for Optoelectronic Applications Physica Status Solidi B 2561800454

[29] Koblmüller G, Pongratz P, Averbeck R, and Riechert H 2002 Delayed nucleation during molecular-beam epitaxial growth of $\mathrm{GaN}$ observed by line-of-sight quadrupole mass spectrometry Applied Physics Letters 80 2281-3

[30] Hestroffer K and Daudin B 2013 A RHEED investigation of self-assembled GaN nanowire nucleation dynamics on bare $\mathrm{Si}$ and on Si covered with a thin AlN buffer layer Physica Status Solidi RRL 7 835-839

[31] Li H, Geelhaar L, Riechert H, and Draxl C 2015 Computing Equilibrium Shapes of Wurtzite Crystals: The Example of GaN Physical Review Letters 115085503 1-5

[32] Kumaresan V, Largeau L, Oehler F, Zhang H, Mauguin O, Glas F, Gogneau N, Tchernycheva M and Harmand J-C 2016 Self-induced growth of vertical GaN nanowires on silica Nanotechnology 27135602

[33] Yadav R, Dixit C K 2017 Synthesis, characterization and prospective applications of nitrogen-doped graphene: A short review Journal of Science:Advanced Materials and Devices 2 141-149

[34] Jeong H M, Lee J W, Shin W H, Choi Y J, Shin H J, Kang J K and Choi J W 2011 NitrogenDoped Graphene for High-Performance Ultracapacitors and the Importance of Nitrogen-Doped Sites at Basal Planes Nano Letters 11 2472-2477 